\title{
A Subcutaneous Biochip for Remote Monitoring of Human Metabolism: Packaging and Biocompatibility Assessment
}

Andrea Cavallini, Tanja Rezzonico Jost, Seyedeh Sara Ghoreishizadeh, Jacopo Olivo, Maaike Op de Beeck, Benjamin Gorissen, Fabio Grassi, Giovanni De Micheli, Fellow, IEEE, and Sandro Carrara, Senior Member, IEEE

\begin{abstract}
This paper represents the extended version of the conference paper "Developing highly-integrated subcutaneous biochips for remote monitoring of human metabolism" presented at the IEEE Sensors Conference 2012, and presents data on assembly, packaging and short term in vitro and in vivo biocompatibility evaluation of a fully implantable biosensor array. The device was realized integrating three building blocks: 1) a multielectrode platform; 2) an inductive coil; and 3 ) an integrated circuit. The entire system measures $2.2 \mathrm{~mm} \times 2.2$ $\mathbf{m m} \times 15 \mathrm{~mm}$. Corrosion of electronic components and leaking of potentially hazardous substances in the body is prevented with a conformal coating of Parylene $C$, while an outer package of medical grade silicone was employed to create a soft shell suitable for implantation. Biocompatibility experiments did not show in vitro cytotoxicity in the considered period of 7 days, while comparison between 7 and 30 days in vivo implantations showed significant reduction of the inflammatory response in time, suggesting normal host recovery.
\end{abstract}

Index Terms-Biosensors, implantable biomedical devices, electronics packaging.

\section{INTRODUCTION}

$\mathbf{C}$ ONTINUOUS monitoring of humans is already in the market for glucose [1] and lactate [2], thanks to the electrochemical sensing, and prototypes of fully implantable

Manuscript received May 11, 2014; accepted July 7, 2014. Date of publication July 18, 2014; date of current version November 7, 2014. This work was supported in part by the SNF Sinergia Project CRSII2 127547/1 entitled Innovative Enabling Micro- Nano-Bio-Technologies for Implantable Systems in Molecular Medicine and Personalized Therapy, the i-IronIC project with a grant from the Swiss Nano- Tera.ch, and evaluated by the SNF and the ERC project, and by the NanoSys project within the program ERC-2009AdG- 246810. The associate editor coordinating the review of this paper and approving it for publication was Dr. Perena I. Gouma.

A. Cavallini, S. S. Ghoreishizadeh, J. Olivo, G. De Micheli, and S. Carrara are with the Integrated System Laboratory, École Polytechnique Fédérale de Lausanne, Lausanne CH-1015, Switzerland (e-mail: cavallini.andrea@gmail.com: seyedehsara.ghoreishizadeh@epfl.ch; jacopo.olivo@epfl.ch; giovanni.demicheli@epfl.ch; sandro.carrara@epfl.ch).

T. R. Jost and F. Grassi are with the Istituto di Ricerca in Biomedicina, Bellinzona 6500, Switzerland (e-mail: tanja.rezzonico@irb.unisi.ch; fabio.grassi@irb.unisi.ch).

M. Op de Beeck is with the Heterogeneous Integrated Microsystems Department, imec, Leuven 3000, Belgium (e-mail: opdebeem@imec.be).

B. Gorissen is with the Machine Design and Automation Section, Katholieke Universiteit Leuven, Leuven 3000, Belgium (e-mail: benjamin.gorissen@mech.kuleuven.be).

Color versions of one or more of the figures in this paper are available online at http://ieeexplore.iee.org.

Digital Object Identifier 10.1109/JSEN.2014.2339638

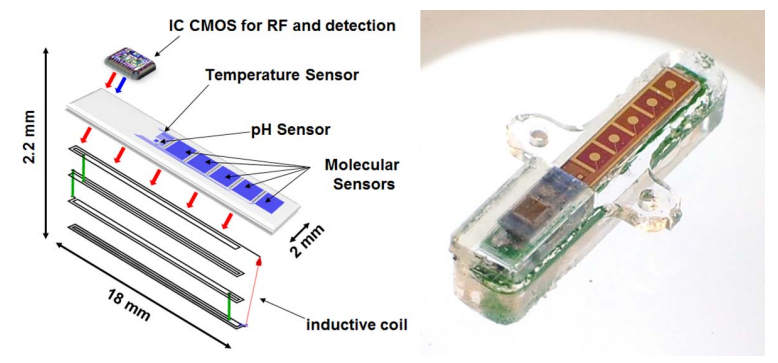

Fig. 1. The fully implantable platform. Left: assembly schematics; right, packaged device.

glucose sensors have been validated up to 8 month in mice [3] and up to one year in pigs [4]. The next step will be the extension of this technology to other relevant metabolites such as, glutamate [5], ATP [6] and drugs [7], [8]. An open challenge is the integration of all these metabolites in a subcutaneous biosensor array capable to provide minimally invasive human telemetry. Such system must satisfy several requirements: sensitivity, as the operative concentration of the biosensor must correspond to the therapeutic range of the target compound in the body; specificity, as the sensors must be able to operate in complex solutions like plasma or interstitial fluid without detecting any interferant; autonomy, as the device must be capable to perform automatically the measurements without the need of external input and without being subjected to power shortages; biostability, as the device should remain functional after the insertion; biocompatibility, because the implant must be well tolerated by the host and cause a limited foreign body reaction;

In this paper we present a prototype of a fully implantable device based on three building blocks: a passive chip hosting 5 independent biosensor electrodes, a temperature and a $\mathrm{pH}$ sensor; an inductive coil for the remote powering of the sensor towards an external wearable device, and an integrated circuit performing the electrochemical measurements (fig. 1). Such device represents a novelty compared to the existing implantable sensors, measuring a single compound, battery powered, and bearing a hard packaging. The device sensitivity can be promoted by nanostructuring the electrode surface with carbon nanotubes (CNT). Although the in-vivo application of CNT is controversial in healthcare applications due to discordant results about their safety [9], the addition of CNT 
proved to be essential to detect physiological concentrations of analytes in human plasma, as demonstrated by our previous works [7], [8]. Furthermore, various measures are taken to prevent CNT release from the implant. The presence of multiple sensors is a strategy to achieve specificity in electrochemical drug detection with P450 biosensors: cytochromes p450 have a broad substrate range and atypical kinetics. Drugs are detected by cyclic voltammetry, and recognized by their specific electrochemical signature, a characteristic potential shift of the p450 reduction peak [10]. The presence of different biosensors specific for the same target, combined with a temperature sensor and a $\mathrm{pH}$ sensor, enables the correct interpretation of the electrochemical signature of the analytes [11], [12]. A battery-less system for the remote powering of the device [13], and an integrated circuit capable of generating on-board voltage ramps [14], ensure the device autonomy; finally, to promote biostability and biocompatibility, we added a double protection for electrodes and electronic components: enzymes and CNTs were entrapped in a chitosan (CHT) matrix and then sealed behind a porous polycarbonate membrane. Chitosan is a natural polysaccharide with unique biological properties including non-toxicity, physiological inertness, affinity to proteins, hemostatic fungistatic and antitumoral properties, which already found employ in biosensors [15], while polycarbonate membranes are commonly employed as microdialysis filters in commercial biosensors [1]. Corrosion of electronic components and leaking of potentially hazardous substances in the body was prevented by a conformal coating of Parylene C, an inert and biocompatible polymer with widespread industrial use as diffusion barrier. An outer package of medical grade silicone was then employed to create a soft shell suitable for implantation. The present work, which represent an extension of the conference paper "Developing highlyintegrated subcutaneous biochips for remote monitoring of human metabolism" [16], presented at the conference IEEE Sensors 2012, focus on the packaging and the short-term biocompatibility evaluation of our implantable device. The efficacy of the parylene $\mathrm{C}$ barrier, as well as the toxicity of carbon nanotubes, have been assessed with a 7-day in-vitro cytotoxicity elution test conform to the ISO-10993-1 standard. The integrity of the CHT/CNT matrix and the efficacy of the polycarbonate membrane in preventing CNT leaking were tested by exposing the materials to solutions of different nature and $\mathrm{pH}$ at $37{ }^{\circ} \mathrm{C}$ for one week. The final packaged device was then implanted in mice for 7 and 30 days to evaluate the inflammatory response.

\section{SYSTEM AND BUILDING BLOCKS DESIGN}

\section{A. Passive Chip}

Choice of materials and design was done considering biocompatibility and simplicity of fabrication as of primary importance. Silicon wafers with 500nm of native oxide were chosen as substrate. Chip metallization was realized by evaporation of $10 \mathrm{~nm}$ of $\mathrm{Ti}$, followed by $100 \mathrm{~nm}$ of Pt. Metal passivation was made via atomic layer deposition of $20 \mathrm{~nm}$ of $\mathrm{Al}_{2} \mathrm{O}_{3}$. Passivation openings were made by dry etching with Argon Ion Milling. Only two masks resulted necessary

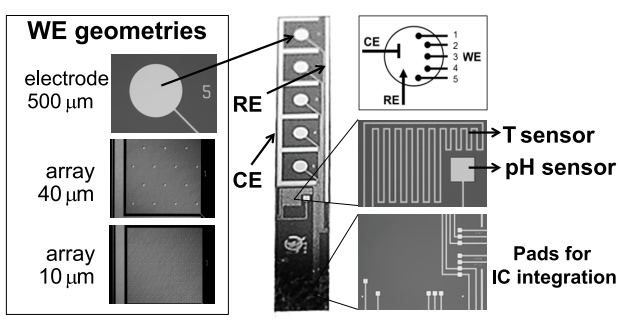

Fig. 2. Photographs of the passive chip. Working electrodes were realized in different geometries: as arrays of 10 and $40 \mu \mathrm{m}$ and as a single electrode of $500 \mu \mathrm{m}$ (left); center whole platform right, temperature and $\mathrm{pH}$ sensors, pads for IC integration and schematics of the electrochemical cell. image from [20].

for the whole microfabrication. As substrate, silicon has been chosen over glass for its better thermal conductibility, in order to improve the dissipation of heat generated by the integrated circuit. Pt metallization was chosen for three main reasons: 1) biocompatibility and resistance to corrosion, 2) pseudoreference electrode behavior, 3) employment in the fabrication of resistive thermal devices with a linear range suitable to measure physiological temperatures [17]. $\mathrm{Al}_{2} \mathrm{O}_{3}$ is a biocompatible material already used in biomedical coatings [18]. Atomic layer deposition was chosen among other techniques for its capability to generate thin, uniform and pinhole free passivations [19]. Figure 2 shows photographs of the passive chip: the platform measures $2.2 \times 15 \mathrm{~mm}$, and host five independent platinum working electrodes (WE) with common reference (RE) and counter (CE) electrodes; a $\mathrm{pH}$ sensor based on an anodic iridium oxide film; a resistive platinum thermal device (Pt RTD) as temperature sensor, and pads for the wire bonding of the integrated circuit. The inclusion of common reference and counter electrode allowed simplifying the interconnections with the sensing circuitry, optimizing at the same time the available space. The working electrodes can be individually functionalized with high precision towards a single-step electrodeposition of a solution containing CHT $0.7 \% \mathrm{w} / \mathrm{v}$; MWCNT $1 \%$ and the enzyme at an appropriate working concentration (i.e. $15 \mathrm{mg} / \mathrm{ml}$ ). The polarization of an electrode at $+1.5 \mathrm{mV}$ for at least 300 " creates a localized region of high $\mathrm{pH}$ that can exceed chitosans solubility limit, allowing CHT polymerization and the entrapment of any other compound present in the original solution with high spatial selectivity, while variations in the electrodeposition time allow to control the amount of material deposited. Characterization of the electrodes, biosensors, electrodepositon, $\mathrm{pH}$ and temperature sensors is extensively reported in [20].

\section{B. Measurement Circuit}

In order to integrate sensors and electronics into a single device, the front-end electronics for the realization of the two mostly used detection techniques, Chronoamperometry (CA) and Cyclic Voltammetry (CV), must be carefully designed. At present, there is little literature concerning the integration of the waveform generator to the implantable biochip which is essential for a fully-integrated CV measurement [21], [22], and most of the designed electronics for the biosensors use an external generator. Li et al. described a ramp generator circuit 


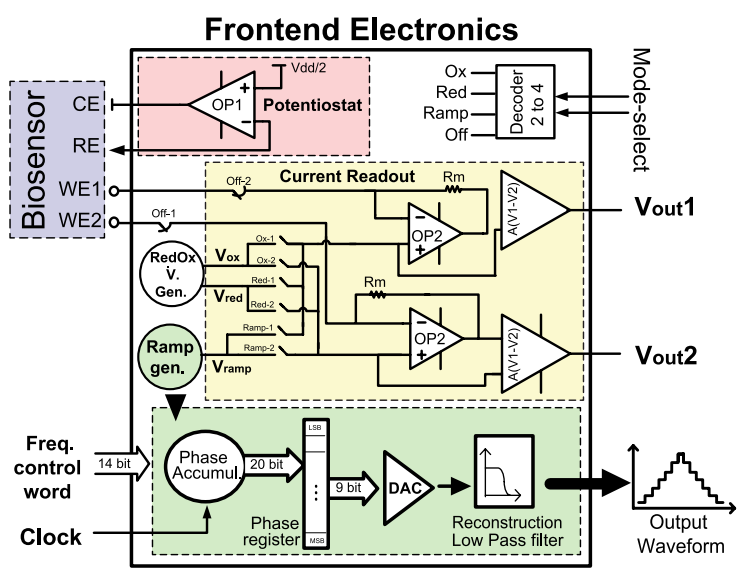

Fig. 3. Proposed architecture for the frontend electronics.

that can be integrated in a biosensor array [23]; however the system presented has limited versatility, since all the electrodes in the array are subjected to the same potential. In our work the $\mathrm{CE}$ and RE are shared while each WE is designed to host different biosensors requiring different potentials, or even different methods to be activated. For this reason, a specific voltage, either fixed or varied in time, must be applied to each WE to enable the sensing of the various compounds. Fig. 3 shows the architecture of the proposed frontend electronics, which consists of three main parts: 1) a ramp generator circuit, 2) a potentiostat, and 3) a current readout circuit. The ramp generator produces a very-low slope and a low-frequency ramp voltage, and is capable to generate a triangular waveform with a slope adjustable from less than $10 \mathrm{mV} / \mathrm{sec}$ to more than $100 \mathrm{mV} / \mathrm{sec}$ with a rail-to-rail swing. The steps are $3.3 \mathrm{mV}$ and the size of the circuit is $0.122 \mathrm{~mm}^{2}$ [24]. The circuit is based on the Direct Digital Synthesis method (DDS), which includes a numerically controlled oscillator and a Digital-toAnalog Converter (DAC). The oscillator produces a quantized version of the desired waveform, whose period is controlled by the digital word contained in the Frequency-Control-Register (FCR). The resulting waveform is then converted to analog by the DAC [24].

The circuit has been implemented in $0.18 \mu \mathrm{m}$ technology. Simulations are done using the electrical equivalent model of the biosensor from [25]. Simulation results show the readout circuit senses currents in the range of $+/-5 \mu \mathrm{A}$ with an equivalent input referred current noise of $3 \mathrm{nA}$ rms. Highly controllable low triangular waveform is also achieved. Further simulation and measurement results on the measurement circuit are reported in [14], [24], and [26]. The maximum power consumption of the measurement circuit is $530 \mu \mathrm{W}$, a value suitable for the remote powering of our device.

\section{Receiving Coil}

Remote powering through inductive link is one of the most promising approaches to supply power to implantable devices, and the miniaturization of power-efficient inductors, is still an open topic. To this end, we propose an implantable coil based on the multi-layer approach. Such strategy, already exploited in integrated circuits [27], [28], it is not yet commonly used on printed circuit boards (PCB). In a multi-layer coil, a spiral inductor is replicated on the two layers of different PCBs. The boards are then stacked, and the inductors are electrically connected as shown in fig. 1 . Our coil was obtained by using a 12-layers, 21-turns inductor of $30 \mathrm{~mm}$ with a thickness of $816.4 \mu \mathrm{m}$. Due to the higher number of turns, our coil has an improved coupling with the external receiver when compared to a single-layer coil of equivalent surface [29]. The system also performs bidirectional data communication without any implanted RF transmitter: downlink communication is obtained via Amplitude Shift Keying (ASK), while uplink communication exploits a backscattering technique. In backscattering, the internal load is modulated according to the outgoing bit-stream; this change is detected by the external part as a variation of the current flowing on the external inductor. A high efficiency class-E power amplifier was utilized to drive the external inductor. Powered by two thin lithium-ion polymer batteries, the system can transfer up to $15 \mathrm{~mW}$ over a distance of $6 \mathrm{~mm}$ in air. The maximum link efficiency measured was $13 \%$. Furthermore, the system can transfer up to $1.17 \mathrm{~mW}$ when a $17 \mathrm{~mm}$ beef sirloin is placed between the inductors, which is enough power for the measurement circuit.

\section{BIOCOMPATIBILITY ASSESSMENT - MATERIALS AND METHODS}

\section{A. Matrix Corrosion Test}

CHT/CNT dispersion was prepared according to [6]. Drops of $10 \mu \mathrm{l}$ were cast onto a 12 well plate and dried under laminar flow. Half of the samples were protected with a polycarbonate membrane (Cyclopore track etched membrane, cut off 100nm, Whatman) and sealed with fast curing medical grade silicone (Med2-4220, Nusil). All samples were covered with $1 \mathrm{ml}$ solutions of milliQ water, PBS $1 \times \mathrm{pH} 4$, or Mouse Embryonic Fibroblasts (MEF) growth media, and put in a cell incubator at $37{ }^{\circ} \mathrm{C}\left(5 \% \mathrm{CO}_{2}\right)$ for 7 days. The $8^{\text {th }}$ day the solutions were removed and the samples rinsed twice with DI water before being dried in air under laminar flow. The CHT/CNT matrix integrity and the dispersion of CNT aggregates on the well plate surface were then inspected with an optical microscope.

\section{B. In-Vitro Cytotoxicity Test}

Preparation of Contaminated Medium: The growth medium was obtained adding to a Dulbecco Modified Eagle Medium, Fetal Calf Serum (10\% v/v); L-glutamine $200 \mathrm{mM}(1 \% \mathrm{v} / \mathrm{v})$; Glutamax (2\% v/v); non-essential aminoacids (1\% v/v), Penicillin/Streptavidin $(1 \% \mathrm{v} / \mathrm{v})$. All the reagents were purchased from Gibco. Test materials were placed in cell culture dishes, sterilized with ethanol $70 \%$ and dried under laminar flow. MEF medium was added according to the ratio $1 \mathrm{ml} / 6 \mathrm{~cm}^{2}$ of material. The samples were put in a cell incubator at $37{ }^{\circ} \mathrm{C}, 5 \% \mathrm{CO}_{2}$ for 7 days. A negative control of fresh, uncontaminated MEF medium was also included to account for the aging of nutrients in solution. The contaminated medium and the control were then collected, stored at $4{ }^{\circ} \mathrm{C}$ and used within 3 days. Cell culture: Mouse Embryonic 
Fibroblasts (MEF) were extracted, seeded in 12-well plates at the concentration of $1.2 \times 10^{4}$ cells $/ \mathrm{ml}$ and grown for 3 days. The $3^{t} h$ day, the growth medium was collected and replaced with the contaminated terrain and the control. Cells were grown for 4 additional days before being inspected for viability and toxicity. Assessment of Viability: The specimens were rejected when the viability of healthy control cells resulted less than 75\% [30]. Control wells were incubated 30 with $1 \mu \mathrm{g} / \mathrm{ml}$ of DAPI and PI fluorescent dyes (Invitrogen) to highlight respectively total cell nuclei and dead cells. For each well, three DAPI and PI counts in different spots were performed. Cell viability was then calculated according to the equation

$$
\text { viability }=\frac{\text { viable cells }}{\text { total cells }} 100 \%
$$

where viable cells is the total number of DAPI counts minus PI counts and total cells is total number of DAPI counts.

Toxicity Evaluation: Cells were incubated 30 with $1 \mu \mathrm{g} / \mathrm{ml}$ of Calcein AM (Invitrogen). The fluorescence in each sample was immediately measured with a commercial scanner (Tecan infinite M1000, excitation $\lambda 485 \mathrm{~nm}$, emission $525 \mathrm{~nm}$ ). The total fluorescence count for each well was obtained averaging 225 readings evenly distributed in a $15 \times 15$ round shape along the well area. Since Calcein AM stains only living cells, cytotoxicity was evaluated comparing the fluorescence intensity of the contaminated samples with the control. According to the United States Pharmacopeia (USP) standards, intensity decrease of more than $20 \%$ from the control was considered cytotoxic. Additionally, cells were inspected for morphologic abnormalities by fluorescence microscopy. Samples presenting a majority of abnormal cells with respect to the control were considered cytotoxic.

\section{Assembly and Packaging}

Loctite 3211 USP class VI biocompatible glue was purchased from Loctite. Adhesion promoter Silane A174 was obtained from Merck and applied to the samples according to the procedure described in [31]; Parylene $\mathrm{C}$ was bought from Speciality Coating Systems and deposited by chemical vapor deposition using a Comelec C-30-S Parylene Deposition System. The plexiglass mold for the final silicone encapsulation was realized by micromachining. Biocompatible medicalgrade silicone (Med-6033) was bought from Nusil.

\section{In-Vivo Biocompatibility}

Microchips were cleaned and disinfected with Gigasept instru AF (Schuelke), placed in cell culture dishes, sterilized with ethanol 70\% and dried under laminar flow. An Air Pouch (AP) was created by subcutaneous injection of sterile air in the back of male C57BL/6 mice at day $1(5 \mathrm{~mL})$ and day $3(3 \mathrm{~mL})$; this procedure creates a cavity of $1.5 \mathrm{~cm}$ diameter and $0.5 \mathrm{~cm}$ height. At day 6, mice were anesthetized with isoflurane 4\%, shaved and locally sterilized with Betadine Solution; the sterile microchips were implanted and the cavity sutured with Vicryl 6.0 (Provet AG). As a control of local inflammation, bacterial lipopolysaccharide (LPS) (50g/mouse) (LabForce AG) was

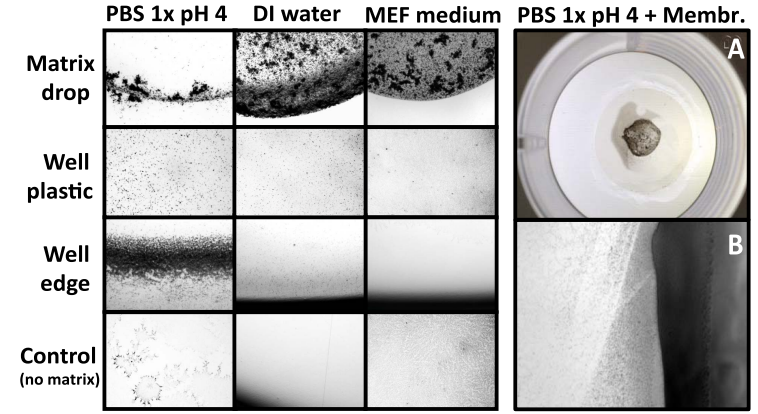

Fig. 4. Corrosion test of the CHT/CNT matrix. Black spots represent chunks of CHT/CNT attached to the surface. A, B evaluation of CHT/CNT leaking from a polycarbonate membrane.

injected daily into the cavity for 4 days or 2 weeks for short term and long term biocompatibility, respectively. As negative control air pouches were generated in the absence of any surgical procedure. After 7 or 30 days, the microchips were removed. The cavity was rinsed with $0.5 \mathrm{~mL}$ of PBS (Gibco) and the liquid collected and centrifuged at 7000rpm for 10' at $4{ }^{\circ} \mathrm{C}$. We then determined the concentration of ATP in the supernatant with ATP detection kit (ATP Determination Kit, Invitrogen). For polymorphonuclear neutrophils detection, the pellet was resuspended in $0.2 \mathrm{~mL}$ RPMI $10 \%$ fetal bovine serum (FBS) (Gibco) and analyzed at flow cytometer (FACS Canto, Becton Dickinson) with antibodies specific for CD11b and Gr1, respectively labeled with allophycocyanin (APC) and fluorescein isothiocyanate (FITC) (both from BioLegend).

\section{Biocompatibility Assessment - Results AND DISCUSSION}

\section{A. Matrix Corrosion Test}

To assess if the CHT/CNT matrix represent a good strategy for enzyme immobilization in implantable sensors, we tested its resistance to corrosion in different solutions: PBS $1 \times \mathrm{pH} 4$, distilled (DI) water, and MEF medium after 7 days of incubation at $37{ }^{\circ} \mathrm{C}\left(5 \% \mathrm{CO}_{2}\right)$ (fig. 4). Data shows at acidic $\mathrm{pH}$ $\mathrm{CHT} / \mathrm{CNT}$ aggregates are spread on the well surface and tend to accumulate at the well edge. The extent of matrix corrosion is due to the different solutions $\mathrm{pH}$ : chitosan has a $\mathrm{pK}_{a}$ of $\cong 6.5$. When the solution $\mathrm{pH}$ is lower than chitosan $\mathrm{pK}_{a}$, the amino-groups of chitosan are protonated and the compound becomes water soluble. Differences in corrosion between water and MEF medium are attributed to the buffer properties of the latter: cell substrates are optimized to maintain a physiological $\mathrm{pH}$ during the incubation. In a second experiment we evaluated if the presence of a polycarbonate membrane is sufficient to prevent significant CNT leaking in the body. After 7 days of incubation in PBS $1 \times \mathrm{pH} 4$, the matrix drop is still evident, but a darker area in its proximity denotes the presence of severe corrosion fig. 4 (A). Optical microscope images of the silicone seal edge fig. 4 (B) clearly show that CNTs and chitosan in solution are able to leak from the membrane. 

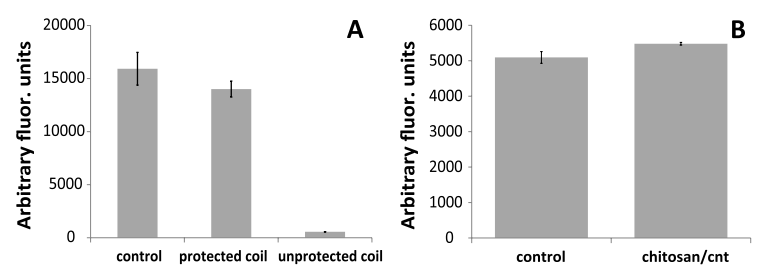

Fig. 5. Evaluation of materials cytotoxicity by calcein staining - average fluorescence values. Error bars, standard deviation $(1 \sigma)$.

\section{B. In-Vitro Citotoxicity Test}

The building blocks of the implantable sensor are partially made from non-biocompatible or potentially toxic materials. For example, the receiving coil presents a copper metallization, notably cytotoxic [32], while materials of the integrated circuit and of the auxiliary electric components, realized by external companies, are not totally disclosed, and have to be considered as potentially harmful. A conformal coating of $3 \mu \mathrm{m}$ parylene $\mathrm{C}$ was employed to prevent both corrosion of the electrical parts and toxic metals leaking in the surrounding tissue. Parylene efficacy was evaluated by elution tests using the copper receiving coil as test substrate. When immersed in a fluid, the copper contained in the coil diffuses in the solution, producing severe cytotoxicity even at very small concentrations. Cytotoxicity was assessed with elution tests according to the ISO 10993-1 guidelines. Each experiment was conducted in triplicate and included a positive control to assure reproducibility and reliability of results. Fig. 5A presents the average fluorescence of Calcein-AM stained cells. After 4 days of incubation with the contaminated medium, cells grown in presence of the terrain eluted with the unprotected coil yielded severe cytoxicity, as demonstrated by very low fluorescence; On the other hand, the parylene $\mathrm{C}$ coating proved effective in preventing severe cytotoxicity, as the fluorescence intensity resulted the $88 \%$ of the intensity of the control (cells grown in uncontaminated medium). It is worth mentioning that while variations up to $20 \%$ from the control are not considered cytotoxic by the USP guidelines, such variability could be unacceptable in long term implants [30]. The protection of the parylene $\mathrm{C}$ coating can be enhanced improving its substrate adhesion and its thickness. Accelerated elution tests performed on our silicon electrodes ( 3 days at $70{ }^{\circ} \mathrm{C}$ in PBS $1 \times \mathrm{pH} 7.4$ ), showed that coatings of $3 \mu \mathrm{m}$ on surfaces not treated with adhesion promoter, tend to form bubbles and eventually detach from the surface (fig. 7). In a second experiment we evaluated the cytotoxicity of the CHT/CNT matrix (fig. 6B). After 7 days of elution and four days of incubation with healthy cells, no significant differences with the control cells have been observed, proving that in the considered period the CNT leaking is not cytotoxic. A possible explanation is that CNT toxicity depends on many factors like length, functionalization or aggregation [9], [33]. Also, the tight wrapping of chitosan to the nanotubes, which is also responsible for their dispersion in aqueous solutions [34], might shield the nanoparticle and its reactive groups, reducing the inherent toxicity and preventing their aggregation. The lack of short-term cytotoxicity of chitosan/CNT nanocomposites is a very promising result towards the employment of this nanomaterial in biomedical

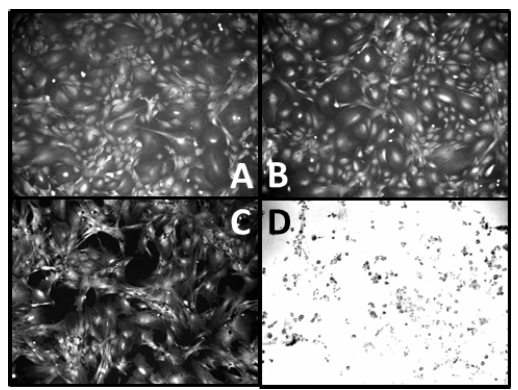

Fig. 6. Morphology of MEF grown in different contaminated media. A) control, B) protected coil, C) CHT/CNT matrix, and D) unprotected coil.

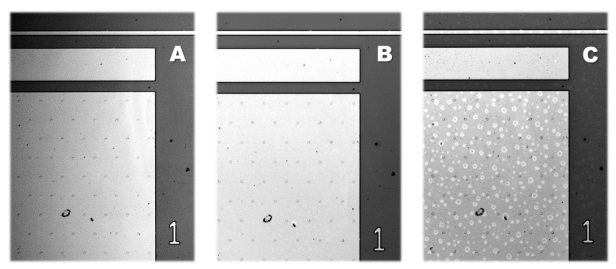

Fig. 7. Accelerated elution test of the sensor platform covered by $3 \mu \mathrm{m}$ of parylene C. Optical microscope images. A) Before the test; B) ater 1 week at $37{ }^{\circ} \mathrm{C}$ in MEF medium; and C) after 3 additional days at $70{ }^{\circ} \mathrm{C}$ in MEF medium.

applications; however, it is important to say that the application of carbon nanotubes in implantable devices relies strongly on their long-term safety: at best of our knowledge, up to date there are no exhaustive studies concerning the longterm effects of functionalized CNT in living organisms or the impact of these substances on the environment. As additional cytotoxicity control we performed a morphological analysis of the cells exposed to the elution fluids. Fig. 6 shows that cells from the protected coil (B) and from the CHT/CNT matrix (C) are similar in shape and distribution to the control (A). No living cells were found on the sample with fluid eluted from the unprotected coil ( $\mathrm{D}$, bright field image). The giant cells shown in the pictures are due to the initial cell density, which after 7 days resulted too low to give a confluent coverage and the typical tight and elongated shape of fibroblasts.

\section{Assembly and Packaging}

The sensor building blocks were glued together using a USP class VI biocompatible glue. Component interconnection was realized with $\mathrm{Al}$ wire bonding and protected with glob top. To further improve parylene $\mathrm{C}$ adhesion and moisture penetration, the assembled platform was treated with silane A-174 and coated with $16 \mu \mathrm{m}$ of parylene C. The outer silicon shell was realized by placing the implant into a plexiglass mold and injecting biocompatible medical-grade silicone. To increase the host comfort, the outer shell was made $1 \mathrm{~mm}$ thick and with rounded corners. In a design variant, two wingsof $3 \times 3 \mathrm{~mm}$ placed along the main body have been included to prevent the sensor to capsize after implantation, since misalignment between the receving coil and the external wearable device can compromise the remote powering of the sensor array. $0.1 \mu 1$ of CHT/CNT suspension was then manually drop cast on the electrodes and dried in air. 


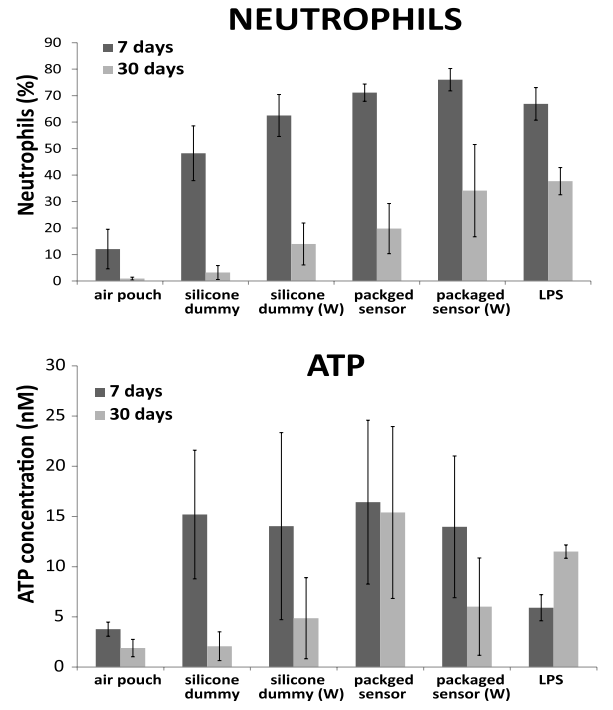

Fig. 8. In-vivo biocompatibility results after 7 and 30 days. Top: neutrophils infiltration in the implant cavity (\%); bottom, ATP concentration. Error bars, standard deviation $(1 \sigma)$.

A polycarbonate membrane was placed above the electrodes and sealed to the external shell using fast curing medical grade silicone. Without considering the external wings, the packaged device measures $20 \times 4.2 \times 3 \mathrm{~mm}$. The final device is shown in fig. 1.

\section{In-Vivo Biocompatibility}

Potential sources of inflammation in implantable devices, can be attributed to the implant materials, shape and dimensions [35]. In order to investigate which elements are critical in eliciting an inflammatory response, we fabricated devices of different nature and shape to subcutaneously implant in mice. To account for inter-individual variability, each model was tested in eight different animals. Half of the mice carried the implant for 7 days, the other half for 30 days. At the end of the period, the implant site was washed with PBS, and levels of ATP and neutrophils in the elution liquid were compared to follow the local inflammatory response. While ATP release is a consequence of cellular necrosis and it is therefore a measure of the local cell damage, neutrophils are recruited to the inflammation site by chemical signaling. Variations in neutrophil percentage at the implant site are therefore informative of the status of tissue inflammation [36]. Fig. 8 presents neutrophils and ATP variation in the liquid collected from the implant site after 7 and 30 days. Sensors packaged with a soft shell with or without external wings (W), were compared with dummy package replicas entirely made in biocompatible silicone. Bacterial lipopolysaccharide (LPS) was administered to a separate group of mice in order to induce an inflammatory response not caused by an artificial implant (positive control), while mice with air pouch only served as negative control.

After 7 days, we measured high levels of ATP and neutrophils, but both values substantially decreased after 30 days in a way proportional to the insert complexity (presence of wings and/or sensors), suggesting that the organism became tolerant to the implants. Data from neutrophils suggests that the insert complexity (presence of wings and/or sensors) tends to increase the short-term and long-term inflammatory response. Considering average values, after 30 days, residual neutrophils in mice treated with winged inserts were 2-3 folds higher than their counterparts, while the presence of the sensor platform induced slightly higher inflammatory responses. ATP measurements in mice bearing the implant were affected by large statistical variability. A possible explanation is that upon removal we found that several implants were displaced from their original location. This may have led to internal tissue damage due to mechanical friction, and therefore insurgence of cellular damage and ATP release in some individuals [37]. Unfortunately, this large variation in the ATP measurements makes difficult to establish a clear relationship between implant complexity and extent of local cell death.

Values from LPS injection further suggest how after 30 days the host seems to accept the insert. Although LPS-induced ATP and neutrophils were detected in lower amounts compared to the values obtained from implants, ATP concentration in animals treated with LPS almost doubled after 30 days. The pyrogenic effect of LPS is also evident with respect to the neutrophils infiltration: in one month, LPS-treated mice presented the highest percentage of neutrophils and the slightest timedependent reduction among the animals considered. From the neutrophils measurements it appears that the package shape has a role in determining the inflammatory response, as the presence of wings tends to increase the immune reaction. Although the introduction of stitching wings may be necessary to hold the insert in place and correctly aligned with the external powering coil, their presence can exert an uncomfortable localized pressure on some parts of the tissue, and angles where tissue stress might promote localized immune responses [38]. The presence of the electronic platform further increased the inflammation response. However, it is important to say that more than strong conclusions, these are trends suggested by the average values obtained. In-vivo experiments tend to have large variability, and due to the reduced number of animals used, the overlap of error bars indicates that results are not statistically different. A larger number of experiments, or different investigation techniques are advised for a more precise evaluation of the in-vivo biocompatibility. The removal of copper components from the sensor electronics, together with thicker parylene coatings may further reduce the inflammation caused by the biosensor platform. The reduction of implant dimensions may also help increasing the host tolerance. In this respect, the bulkiest component of our platformis the inductive coil, which with a thickness of $2 \mathrm{~mm}$, contributes to almost $4 / 5$ of the total platform volume. Our group is currently developing a single layer coil on silicon, which will reduce the platform thickness to $11.5 \mathrm{~mm}$.

\section{CONCLUSION}

This paper represent the extended version of the work descrived in reference [16], and presents a novel, highly integrated system for human metabolism telemetry, focusing 
on its packaging and biocompatibility. The system is made of three parts: a passive chip hosting 5 independent biosensors, a $\mathrm{pH}$ sensor and a temperature sensor; a CMOS integrated circuit capable to perform the required electrochemical measurements, and a multi-layer antenna for the remote powering of the device and the transmission of the sensing data. The electrodes of the passive chip can be functionalized with proper bio and nanostructures with high spatial precision towards electrodeposition of a solution of chitosan, carbon nanotubes and enzymes [16]. The CMOS IC showed good performance in simulations and measurements [24], [26], [39], while the receiving antenna resulted capable to collect enough energy to support the work of the IC [16]. The assembled system was packaged with an inner barrier of parylene $\mathrm{C}$, an outer shell of biocompatible silicone and a polycarbonate membrane to protect the sensor area. The biocompatibility of the device was then tested in-vitro and in-vivo.

In-vitro experiments on primary fibroblasts proved that coatings of $3 \mathrm{M}$ of parylene $\mathrm{C}$ are effective in preventing copper leaking and cytoxicity for at least seven days; similar test on a chitosan/MWCNT matrix showed that release of chitosan/MWCNT complexes in the growth terrain are not cytotoxic in the short term. This last result is in agreement with recent studies demonstrating that the employ of short, functionalized CNT immobilized in a nanocomposite may be implemented with a certain degree of safety. Although, in last analysis, the fate of nanotubes in implantable devices is still largely dependent by studies concerning their long-term toxicity. In-vivo tests of the packaged sensor array demonstrated that the foreign body reaction significantly decreased after 30 days, suggesting normal recovery of the host. Package shape is suspected to have a role in inducing the inflammatory response, suggesting that future implants must possess smoother and simpler geometries.

Taken all together, these results demonstrate the feasibility of the entire system. Future works will be directed in assessing the performance of the fully implantable system in autonomous measurements in-vitro of different metabolites and in promoting the long-term biocompatibility and biostability of the device.

\section{ACKNOWLEDGMENTS}

J. O'Callaghan, K. Qian, and T. Miyazaki are acknowledged for the support and supervision during the in-vitro biocompatibility tests at Imec, Belgium. D. Sacchetto is acknowledged for the advices on the microfabrication of the passive chip and M. De Marchi and C. Baj-Rossi for their help in formatting the text.

\section{REFERENCES}

[1] F. Valgimigli, F. Lucarelli, C. Scuffi, S. Morandi, and I. Sposato, "Evaluating the clinical accuracy of GlucoMen day: A novel microdialysisbased continuous glucose monitor," J. Diabetes Sci. Technol., vol. 4, no. 5, pp. 1182-1192, 2010.

[2] A. Poscia, D. Messeri, D. Moscone, F. Ricci, and F. Valgimigli, "A novel continuous subcutaneous lactate monitoring system," Biosensors Bioelectron., vol. 20, no. 11, pp. 2244-2250, 2005.
[3] B. Yu, N. Long, Y. Moussy, and F. Moussy, "A long-term flexible minimally-invasive implantable glucose biosensor based on an epoxyenhanced polyurethane membrane," Biosensors Bioelectron., vol. 21, no. 12, pp. 2275-2282, 2006.

[4] D. A. Gough, L. S. Kumosa, T. L. Routh, J. T. Lin, and J. Y. Lucisano, "Function of an implanted tissue glucose sensor for more than 1 year in animals," Sci. Translational Med., vol. 2, no. 42, pp. 42ra53-42ra53, 2010.

[5] S. Carrara et al., "Single-metabolite bio-nano-sensors and system for remote monitoring in animal models," in Proc. IEEE Sensors, Oct. 2011, pp. 716-719.

[6] A. Cavallini, G. De Micheli, and S. Carrara, "Comparison of three methods of biocompatible multi-walled carbon nanotubes confinement for the development of implantable amperometric adenosine-5'-triphosphate biosensors," Sensor Lett., vol. 9, no. 5, pp. 1838-1844, 2011.

[7] S. Carrara, A. Cavallini, V. Erokhin, G. Albini, and G. De Micheli, "Multi-panel drugs detection in human serum for personalized therapy," Biosensors Bioelectron., vol. 26, no. 9, pp. 3914-3919, 2011.

[8] A. Cavallini, S. Carrara, G. De Micheli, and V. Erokhin, "P450-mediated electrochemical sensing of drugs in human plasma for personalized therapy," in Proc. Conf. Ph.D. Res. Microelectron. Electron. (PRIME), Jul. 2010, pp. 1-4.

[9] S. K. Smart, A. I. Cassady, G. Q. Lu, and D. J. Martin, "The biocompatibility of carbon nanotubes," Carbon, vol. 44, no. 6, pp. 1034-1047, 2006.

[10] D. L. Johnson, B. C. Lewis, D. J. Elliot, J. O. Miners, and L. L. Martin, "Electrochemical characterisation of the human cytochrome p450 cyp2c9," Biochem. Pharmacol., vol. 69, no. 10, pp. 1533-1541, 2005.

[11] S. Carrara, A. Cavallini, A. Garg, and G. De Micheli, "Dynamical spot queries to improve specificity in p450s based multi-drugs monitoring," in Proc. Int. Conf. Complex Med. Eng. (ICME), Apr. 2009, pp. 1-6.

[12] S. Carrara, M. D. Torre, A. Cavallini, D. De Venuto, and G. De Micheli, "Multiplexing $\mathrm{pH}$ and temperature in a molecular biosensor," in Proc. IEEE Biomed. Circuits Syst. Conf. (BioCAS), Nov. 2010, pp. 146-149.

[13] J. Olivo, S. Carrara, and G. De Micheli, "A study of multi-layer spiral inductors for remote powering of implantable sensors," IEEE Trans. Biomed. Circuits Syst., vol. 7, no. 4, pp. 536-547, Aug. 2013.

[14] S. S. Ghoreishizadeh, S. Carrara, and G. De Micheli, "Circuit design for human metabolites biochip," in Proc. IEEE Biomed. Circuits Syst. Conf. (BioCAS), Nov. 2011, pp. 460-463.

[15] B. Krajewska, "Application of chitin-and chitosan-based materials for enzyme immobilizations: A review," Enzyme Microbial Technol., vol. 35, no. 2, pp. 126-139, 2004.

[16] S. Carrara, A. Cavallini, S. Ghoreishizadeh, J. Olivo, and G. De Micheli, "Developing highly-integrated subcutaneous biochips for remote monitoring of human metabolism," in Proc. IEEE Sensors, Oct. 2012, pp. 1-4.

[17] P. R. N. Childs, J. R. Greenwood, and C. A. Long, "Review of temperature measurement," Rev. Sci. Instrum., vol. 71, no. 8, pp. 2959-2978, Aug. 2000.

[18] T. V. Thamaraiselvi and S. Rajeswari, "Biological evaluation of bioceramic materials-a review," Carbon, vol. 24, no. 31, p. 172, 2004.

[19] M. Knez, K. Nielsch, and L. Niinistö, "Synthesis and surface engineering of complex nanostructures by atomic layer deposition," Adv. Mater., vol. 19, no. 21, pp. 3425-3438, 2007.

[20] A. Cavallini, C. Baj-Rossi, S. Ghoreishizadeh, G. De Micheli, and S. Carrara, "Design, fabrication, and test of a sensor array for perspective biosensing in chronic pathologies," in Proc. IEEE Biomed. Circuits Syst. Conf. (BioCAS), Nov. 2012, pp. 124-127.

[21] M. Kimura, H. Fukushima, Y. Sagawa, K. Setsu, H. Hara, and S. Inoue, "An integrated potentiostat with an electrochemical cell using thin-film transistors," IEEE Trans. Electron Devices, vol. 56, no. 9, pp. 2114-2119, Sep. 2009.

[22] J. Zhang, Y. Huang, N. Trombly, C. Yang, and A. Mason, "Electrochemical array microsystem with integrated potentiostat," in Proc. IEEE Sensors, Oct./Nov. 2005.

[23] L. Li, W. A. Qureshi, X. Liu, and A. J. Mason, "Amperometric instrumentation system with on-chip electrode array for biosensor application," in Proc. IEEE Biomed. Circuits Syst. Conf. (BioCAS), Nov. 2010, pp. 294-297.

[24] S. S. Ghoreishizadeh, C. Baj-Rossi, S. Carrara, and G. De Micheli, "Nano-sensor and circuit design for anti-cancer drug detection," in Proc. IEEE/NIH Life Sci. Syst. Appl. Workshop (LiSSA), Apr. 2011, pp. 28-33.

[25] M. M. Ahmadi and G. A. Jullien, "A wireless-implantable microsystem for continuous blood glucose monitoring," IEEE Trans. Biomed. Circuits Syst., vol. 3, no. 3, pp. 169-180, Jun. 2009. 
[26] S. S. Ghoreishizadeh, I. Taurino, S. Carrara, and G. De Micheli, "A current-mode potentiostat for multi-target detection tested with different lactate biosensors," in Proc. IEEE Biomed. Circuits Syst. Conf. (BioCAS), Nov. 2012, pp. 128-131.

[27] J.-Y. Xie, W.-Y. Yin, J. Shi, K. Kang, and Z. D. Chen, "Characterization of on-chip miniature multi-layer spiral inductors for RFICs," Microw. Opt. Technol. Lett., vol. 49, no. 12, pp. 2932-2936, 2007.

[28] A. Zolfaghari, A. Chan, and B. Razavi, "Stacked inductors and transformers in CMOS technology," IEEE J. Solid-State Circuits, vol. 36, no. 4, pp. 620-628, Apr. 2001.

[29] J. Olivo, S. Carrara, and G. De Micheli, "Modeling of printed spiral inductors for remote powering of implantable biosensors," in Proc. 5th Int. Symp. Med. Inform. Commun. Technol. (ISMICT), Mar. 2011, pp. 29-32.

[30] M. O. de Beeck, K. Qian, P. Fiorini, K. Malachowski, and C. Van Hoof, "Design and characterization of a biocompatible packaging concept for implantable electronic devices," J. Microelectron. Electron. Packag., vol. 9, no. 1, pp. 43-50, 2012.

[31] C. Hassler, R. P. von Metzen, P. Ruther, and T. Stieglitz, "Characterization of parylene $\mathrm{C}$ as an encapsulation material for implanted neural prostheses," J. Biomed. Mater. Res. B, Appl. Biomater., vol. 93, no. 1, pp. 266-274, 2010.

[32] M. E. Letelier et al., "Possible mechanisms underlying copper-induced damage in biological membranes leading to cellular toxicity," Chem.Biol. Interactions, vol. 151, no. 2, pp. 71-82, 2005.

[33] M. A. Hussain, M. A. Kabir, and A. K. Sood, "On the cytotoxicity of carbon nanotubes," Current Sci., vol. 96, no. 5, pp. 664-673, 2009.

[34] T. Takahashi, C. R. Luculescu, K. Uchida, T. Ishii, and H. Yajima, "Dispersion behavior and spectroscopic properties of single-walled carbon nanotubes in chitosan acidic aqueous solutions," Chem. Lett., vol. 34, no. 11, pp. 1516-1517, 2005.

[35] S. Arens, U. Schlegel, G. Printzen, W. J. Ziegler, S. M. Perren, and M. Hansis, "Influence of materials for fixation implants on local infection an experimental study of steel versus titanium DCP in rabbits," J. Bone Joint Surgery, Brit. Vol., vol. 78, no. 4, pp. 647-651, 1996.

[36] G. B. Ryan and G. Majno, "Acute inflammation. A review," Amer. J. Pathol., vol. 86, no. 1, p. 183, 1977.

[37] P. Bodin and G. Burnstock, "Increased release of ATP from endothelial cells during acute inflammation," Inflammation Res., vol. 47, no. 8, pp. 351-354, 1998.

[38] G. Melcher, B. Claudi, U. Schlegel, S. M. Perren, G. Printzen, and J. Munzinger, "Influence of type of medullary nail on the development of local infection. An experimental study of solid and slotted nails in rabbits," J. Bone Joint Surgery, Brit. Vol., vol. 76, no. 6, pp. 955-959, 1994.

[39] S. S. Ghoreishizadeh, C. Baj-Rossi, A. Cavallini, S. Carrara, and G. De Micheli, "An integrated control and readout circuit for implantable multi-target electrochemical biosensing," IEEE Trans. Biomed. Circuits Syst., to be published, Jun. 2014, doi: 10.1109/TBCAS.2014.2315157.

Andrea Cavallini received the master's (Hons.) degree in biomolecular biotechnology from the University of Bologna, Bologna, Italy, and the Ph.D. degree from École Polytechnique Fédérale de Lausanne, Lausanne, Switzerland, with a focus on the development of implantable biosensors for diagnostic applications. He was a recipient of the Best Poster Award at the Seventh Nanoeurope Symposium in 2009 and the Bronze Leaf Paper Award at the PRIME Conference in 2010.

Tanja Rezzonico Jost received the master's (Hons.) degree in veterinarian biotechnology from the University of Milano, Milan, Italy. She is currently responsible for in-vivo experiments with the T Cell Development Laboratory, Institute for Research in Biomedicine, Bellinzona, Switzerland. Her current research interests include modeling human pathological conditions and identifying biomarkers in chronic inflammatory diseases.
Seyedeh Sara Ghoreishizadeh received the M.Sc. degree in microelectronics circuits from the Sharif University of Technology, Tehran, Iran. She is currently pursuing the Ph.D. degree at École Polytechnique Fédérale de Lausanne, Lausanne, Switzerland. Her research focuses on design and implementation of low-power analog mixed-signal IC for electrical readout in implantable multitarget biosensors.

Jacopo Olivo received the master's (Hons.) degree in electrical engineering from the University of Bologna, Bologna, Italy. During the master's project, he was involved in the field of biosensors, by designing and developing an integrable system for electrochemical measurements in point-of-care applications for personalized medicine. He received the Ph.D. degree with a focus on energy scavenging techniques for implantable biosensors.

Maaike Op de Beeck received the Engineering degree and the Ph.D. degree in electronics from the Catholic University of Leuven (KUL), Leuven, Belgium, in 1985 and 1993, respectively. She held several research positions at KUL, at Philips, Amsterdam, The Netherlands, at Mitsubishi Electric, Tokyo, Japan, and since 1992, she has been with IMEC, Leuven. During the first 20 years of her carrier, she specialized in advanced lithography. Since 2007, she has been active in the field of biomedical applications, with a focus on packaging of wearable and implantable devices. She is currently the Program Manager of the HUMAN++ program at IMEC.

Benjamin Gorissen received the M.S. degree in mechanical engineering degree from Katholieke Universiteit Leuven, Leuven, Belgium, in 2010, where he is currently pursuing the Ph.D. degree in mechanical engineering. His current research interests include fluidic microactuation mechanisms and biomedical MEMS.

Fabio Grassi is an Associate Professor of Biology with the Medical School, University of Milan, Milan, Italy. In 2002, he joined IRB as the Head of the $\mathrm{T}$ Cell Development Laboratory. His research is focused on various aspects of $\mathrm{T}$ cell physiology, including protein and membrane trafficking, signal transduction, control of cell growth and intercellular communications during $\mathrm{T}$ cell development, and immunopathological conditions.

Giovanni de Micheli is a Professor and the Director of the Institute of Electrical Engineering and the Integrated Systems Center at École Polytechnique Fédérale de Lausanne, Lausanne, Switzerland, and a Program Leader of the Nano-Tera Program. His current research interests include emerging technologies, NoCs, 3-D integration, and heterogeneous platform design. He is a fellow of the Association for Computing Machinery and a member of the Academia Europea. He was a recipient of the IEEE Emanuel Piore Award in 2003 and the Golden Jubilee Medal from the IEEE CAS Society in 2000.

Sandro Carrara is a Lecturer and Scientist with the École Polytechnique Fédérale de Lausanne, Lausanne, Switzerland, the founder and Editor-inChief of BioNanoScience (Springer), the Topical Editor of the IEEE SENSORS JOURNAL, and an Associate Editor of the IEEE TRANSACTIONS ON BIOMEDiCAL CIRCUITS AND SySTEMS. He is a member of the IEEE Sensors Council, and was a CASS Distinguished Lecturer from 2013 to 2014. His scientific interests are on electrical phenomena of nanobiostructured films, and CMOS design of biochips based on proteins and DNA. He has more then 170 scientific publications (including Top-25 Hottest-Articles in 2004, 2005, 2008, 2009, and two times in 2012) and holds 12 patents. 